Planetary Systems in the Universe - Observation, Formation and Evolution

Proceedings IAU Symposium No. 202, (c)2004 IAU

Alan Penny, Pawel Artymowicz, Anne-Marie Lagrange, 6 Sara Russell, eds.

\title{
Episodes of Emission Lines in the Spectra of Red Giants as Signatures of Remnant Planetary Systems
}

\author{
G. M. Rudnitskij \\ Sternberg Astronomical Institute, Moscow State University, \\ 13 Universitetskij prospekt, Moscow, 119899 Russia
}

\begin{abstract}
When a star with a mass of about 1 solar mass enters the red giant stage of its evolution, the radius of its atmosphere reaches several astronomical units. If the star possessed during its main-sequence life a planetary system, similar to the solar system, the planets will be embedded into a rather dense and hot medium. Effects of a planet revolving around a red giant at a short distance (inside its circumstellar envelope) are discussed. Systematic monitoring of the spectra of red giants may reveal periodicities in the emergence of shock-induced emission lines and thus to detect probable remnant planetary systems around these stars.
\end{abstract}

At the end of its evolution, a solar-type star enters the phase of a red giant. Its radius grows from $\sim 1 R_{\odot}$ to a few $10^{2} R_{\odot}$. The star begins to lose matter at a rate of $10^{-7}-10^{-5} M_{\odot}$ year $^{-1}$. An extensive gas-dust circumstellar envelope forms. The red-giant stage is very short as compared to the main-sequence stage; it takes no more than a few hundred thousand years. The ultimate end is a complete loss of the convective shell, formation of a naked white-dwarf core, surrounded by a planetary nebula.

If the star possessed a planetary system during its main-sequence life, at the red-giant stage the closer-by planets, revolving at $R \sim 1-3$ A.U., will be embedded within the star's atmosphere. The more massive ones of them will probably survive the red-giant phase (Struck-Marcell 1988).

Evolution of a red giant having a compact stellar companion (a brown/black dwarf with a mass of $\gtrsim 0.02 M_{\odot}$ or possibly a neutron star) was analysed in a series of papers on the 'double-core evolution' (Soker 1999 and references therein). The fate of a lower-mass companion [(0.001-0.01) $M_{\odot}-$ a planet], embedded in the atmosphere of a star that has become a red giant, was also considered in a number of works. In particular, Soker (1999) proposed to search for Uranus-Neptune-like planets in planetary nebulae, formed in course of the post-AGB evolution.

A planet orbiting around a $1 M_{\odot}$ star at a distance of 1 A.U. would move at velocity $V_{p} \sim 30 \mathrm{~km} \mathrm{~s}^{-1}$. If the star is a red giant, then the planet is embedded in the star's atmosphere, having a temperature $T \sim 2000 \mathrm{~K}$ and particle number density of $\sim 10^{12}-10^{13} \mathrm{~cm}^{-3}$. The velocity of sound $a_{s}$ there would be about $3.4 \mathrm{~km} \mathrm{~s}^{-1}$. Thus, the planet's motion is supersonic, the Mach number $\mathrm{M}=$ $V_{p} / a_{s}$ being about 9 . This motion is similar to a motion of a large meteoritic body in the Earth's atmosphere (Tsikulin 1969). A strong conical shock wave, ionising gas and heating it to $10,000-15,000 \mathrm{~K}$, is formed. 


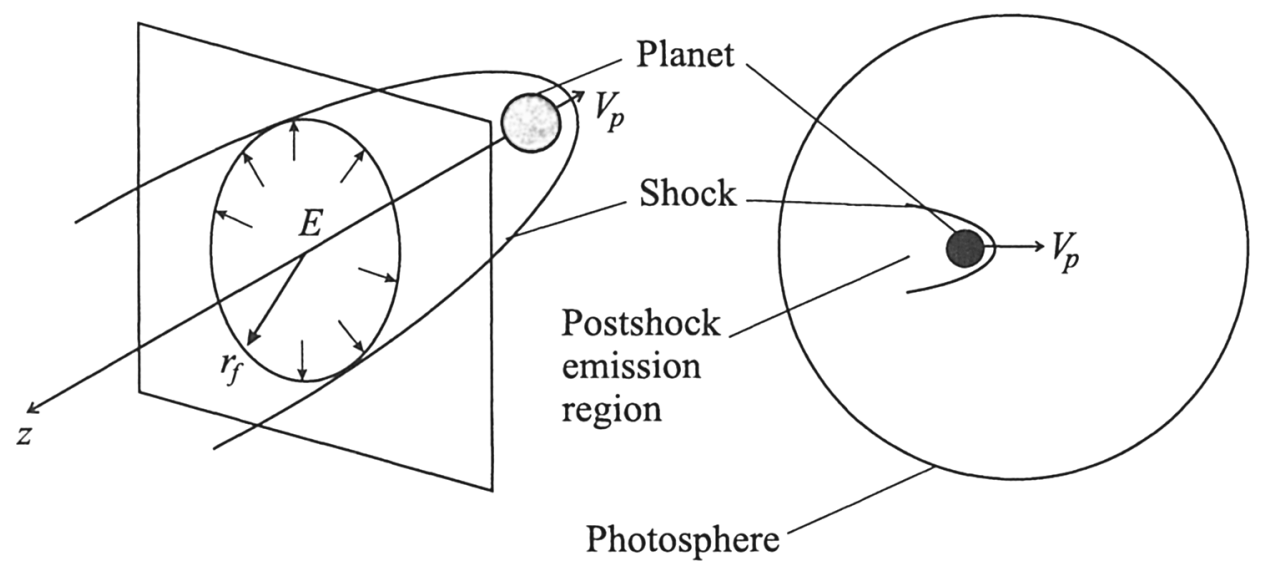

Figure 1. Left: Propagation of a shock wave within a slab perpendicular to the body's velocity vector; $E$ is the energy released in this layer. Right: Motion of a planet across the disc of a red giant at supersonic velocity $V_{p}$.

We consider a simple model, in which a perturbing body with diameter $d$ is moving along a rectilinear trajectory at velocity $V_{p}>a_{s}$ through a medium with mass density $\rho_{0}$. Quantity $E$ is the energy released at a unit path of the body's motion; $E$ is numerically equal to the drag force exerted on the body by the medium:

$$
F=\frac{\pi d^{2}}{4} \rho_{0} V_{p}^{2}
$$

Owing to the drag, the planet is gradually spiraling into the red giant's atmosphere. The rate of decrease of its semimajor axis $a$ is (see, e.g., Taam, Bodenheimer \& Ostriker 1978):

$$
\frac{\dot{a}}{a}=-\frac{F V_{p} a}{G M_{*} M_{p}} .
$$

Using (1) and substituting $a=1$ A.U., $M_{*}=1 M_{\odot}, V_{p}=30 \mathrm{~km} \mathrm{~s}^{-1}$ and Jupiter's parameters $M_{p}=1 M_{\mathrm{J}}=1.9 \times 10^{30} \mathrm{~g}, d=d_{\mathrm{J}}=1.4 \times 10^{10} \mathrm{~cm}$, we have $\dot{a} / a \sim-8 \times 10^{-8}$ year $^{-1}$. Thus, during the red giant stage, which lasts not longer than $10^{6}$ years, the semimajor axis of the planet's orbit decreases by $\$ 8 \%$. For a larger planet $\left(13 M_{\mathrm{J}}, 2.35 d_{\mathrm{J}}\right)$, braking is still smaller, $\lesssim 3.2 \%$.

The motion of the planet through the stellar atmosphere is similar to the case of propagation of a shock wave from a detonating cylindrical charge (Tsikulin 1969). That is, in any plane perpendicular to the trajectory of the body, the propagating shock can be considered as a cylindrical one (see Fig. 1). The shock front radius in this plane is

$$
r_{f}=\left(\frac{E}{\rho_{0}}\right)^{1 / 4} t^{1 / 2}
$$

with $t=z / V_{p}$. The front equation in the $(r, z)$ coordinates (Fig. 1, left) is 


$$
\frac{r_{f}}{d}=\left(\frac{\pi}{4}\right)^{1 / 4}\left(\frac{z}{d}\right)^{1 / 2}
$$

Shock velocity $D$ in the direction perpendicular to the trajectory is decreasing with time as

$$
D=\frac{1}{2}\left(\frac{E}{\rho_{0}}\right)^{1 / 4} t^{-1 / 2}
$$

For a planet with $d=2.35 d_{\mathrm{J}}$, velocity $D$ will fall to the velocity of sound $a_{s}$ at a distance $z_{\max }=d(\pi / 4)^{1 / 2}\left(\mathrm{M}^{2} / 4\right) \sim 5.6 \times 10^{11} \mathrm{~cm}$ behind the body. There will be no emission at greater $z$ 's. The corresponding maximum front radius $r_{f \max }=1.3 \times 10^{11} \mathrm{~cm}$. The maximum projected area of the shocked 'cone' (for a side view, as in Fig. 1, right) is

$$
S_{\mathrm{sh}}=\frac{1}{3}(4 \pi)^{1 / 4} d^{1 / 2} z_{\max }^{3 / 2} \sim 4.8 \times 10^{22} \mathrm{~cm}^{2} \sim 1.6 \times 10^{-5} S_{*},
$$

where $S_{*}=\pi R_{*}^{2} \sim 3 \times 10^{27} \mathrm{~cm}^{2}$ is the stellar disc area for $R_{*} \sim 3 \times 10^{13} \mathrm{~cm}$. Observations and model calculations of the Balmer emission lines in Miras (e.g., Fox \& Wood 1985) show that, for the above-mentioned parameters, the shock front yields up to $10^{20} \mathrm{H} \alpha$ photons $\mathrm{cm}^{-2} \mathrm{~s}^{-1}$; with source area $S_{\mathrm{sh}}$, this can account for the total Balmer line fluxes observed from a star at a distance of about $300 \mathrm{pc}$, a few $\times 10^{-12} \mathrm{erg} \mathrm{cm}^{-2} \mathrm{~s}^{-1}$ (Fox, Wood \& Dopita 1984).

Since 1994, our team has been monitoring a sample of about 20 Miras in the $\mathrm{H} \alpha$ line and in the $\mathrm{H}_{2} \mathrm{O}$ maser line at $\lambda=1.35 \mathrm{~cm}$ (Esipov et al. 1999 and references therein). Some stars ( $\mathrm{R}$ Leo, $\mathrm{R}$ Cas and $\mathrm{U}$ Aur) displayed isolated bursts of the $\mathrm{H} \alpha$ emission, followed (about a year and a half later) by a flare of the $\mathrm{H}_{2} \mathrm{O}$ maser radio emission. This may be due to a periastron shock-wave episode of a planet in a highly eccentric orbit with a period $P \sim 15$ years. Some other stars (e.g., U Ori, Rudnitskij et al. 2000) have already shown some hints to $\mathrm{H}_{2} \mathrm{O}$ maser 'superperiodicity' of 12-15 years - which could be associated with planetary revolution periods.

\section{References}

Esipov, V.F., Pashchenko, M.I., Rudnitskij, G.M., \& Fomin, S.V. 1999, Astron. Lett., 25,672

Fox, M.W. \& Wood, P.R. 1985, ApJ, 297, 455

Fox, M.W., Wood, P.R., \& Dopita, M.A. 1984, ApJ, 286, 337

Rudnitskij, G.M., Lekht, E.E., Mendoza-Torres, J.E., Pashchenko, M.I., \& Berulis, I.I. 2000, A\&AS, 146, 385

Soker, N. 1999, MNRAS, 306, 806

Struck-Marcell, C. 1988, ApJ, 330, 986

Taam, R.E., Bodenheimer, P., \& Ostriker, J.P. 1978, ApJ, 222, 269

Tsikulin, M.A. 1969, Shock Waves Induced in the Atmosphere by Motion of Large Meteoritic Bodies (in Russian) (Moscow: Nauka). 